\title{
On Design and Implementation of Locomotive Video Monitoring System
}

\author{
Haifeng Yuan, Jie Peng \\ Xinjiang Railway Vocational Technical College, Urumqi, Xinjiang, 830011, China
}

Keywords: locomotive video monitoring system, data, video collection, vehicle terminal, monitoring terminal, design

\begin{abstract}
In locomotive operation system, video monitoring system is the most critical. It is responsible for collection, processing and storage of data during operation of locomotive, to ensure safety of locomotive running. Traditional locomotive monitoring management approach has complex operation, but also lacks the intuitive and real-time for locomotive management personnel, which will bring some potential risks for drivers driving. So this paper, based on this situation, designs a new locomotive video monitoring system, adopting data collection, compression, driver-assist, wireless telecommunications and other technologies, to completely enhance the locomotive security monitoring efficiency from vehicle terminal to monitoring terminal.
\end{abstract}

\section{Introduction}

Vehicle terminal and monitoring terminal are the most critical components in locomotive video monitoring system. They assist locomotive drivers to drive and at the same time to achieve ground real-time telecommunication between locomotive and background monitoring center. In terms of operating temperature, electromagnetic environment and internal space and other aspects, achieve the stability and operation convenience of video monitoring system.

\section{Design of locomotive video monitoring system vehicle terminal}

Functions of vehicle terminal designed in this paper cover two parts: assisting driving and data collection and transmission. As locomotive part of locomotive video monitoring system, it uses the embedded design, to save operating space for the small locomotive. Inside, it is not equipped with video collection and compression, data wireless transmission and data operation acquisition modules, etc.

Design analysis of locomotive driver-assist system. Locomotive driver-assist system uses 16-channel video input monitoring system. It is local monitoring, but it is in all parts of the locomotive (cooling room, power room, operating room, etc.). Each local monitoring system can externally connect with 8 large-capacity hard disks, and they will in real time present locomotive local operation conditions they video on LCD screen in driver cab for management personnel. In addition, in order to achieve control of the locomotive driver on camera on the other end of vehicle terminal, hard disk also provides RS485 interface, and it can control hard disk camera through the keyboard, such as camera holder direction control, monitor screen in-turn patrolling and other functional operations. To save application cost, the paper also designs a set of keyboard operating system applicable to hard disk. It uses the MAX485 level switch chip and STX89C51 microcontroller, and its basic structure scheme is shown in Figure 1. 


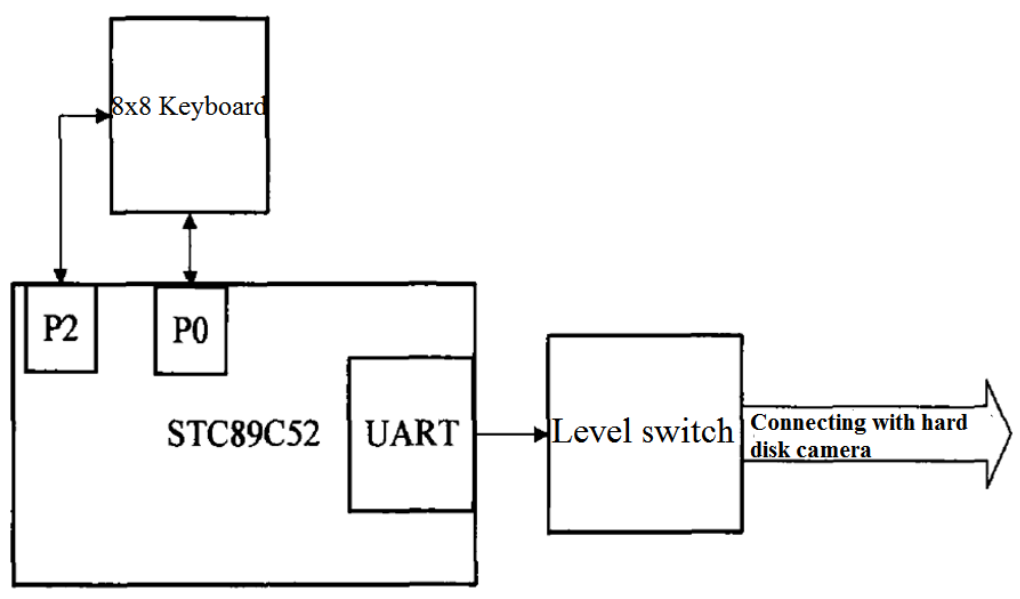

Fig. 1. Hard disk camera keyboard operating system structure

This set of the keyboard operating system design uses a 8x8 determinant key arrangement, able to realize overall operation on hard disk camera, and the operating system uses the line reversal keyboard key scan technology to ensure the normal scan of keyboard program function, avoid operational errors ${ }^{[1] .}$ The following is specific code design of key scan: uint scan_key()

uint key_h,key_1;

$\mathrm{P} 2=0 \mathrm{xff} ; \quad / / \mathrm{P} 2$ row

$\mathrm{P} 0=0 \times 00 ; \quad / / \mathrm{P} 0$ column

if(P2!=0xff)

\{

delay(150);

if(P2!=0xff)

\{

key_h=P2;

$\mathrm{P} 2=0 \mathrm{x} 00 ; \quad / / \mathrm{P} 2$ row row out

$\mathrm{P} 0=0 \mathrm{xff} ; \quad / / \mathrm{P} 0$ column column in

key_1=P0;

return key_h|key_1;

\}

else return 0 ;

\}

Design of data collection and transmission system. Data collection and transmission system of vehicle terminal is wireless communication contact between locomotive and background locomotive management department, able to realize remote monitoring on locomotive. It is the core part of locomotive video monitoring system. Data collection and transmission system is mainly composed of 
the video data collection and compression, locomotive operation data collection and wireless data transmission, which are responsible for all wireless contact and monitoring mission of vehicle terminal video monitoring system. Figure 2 is hardware configuration flowchart of data collection and transmission system.

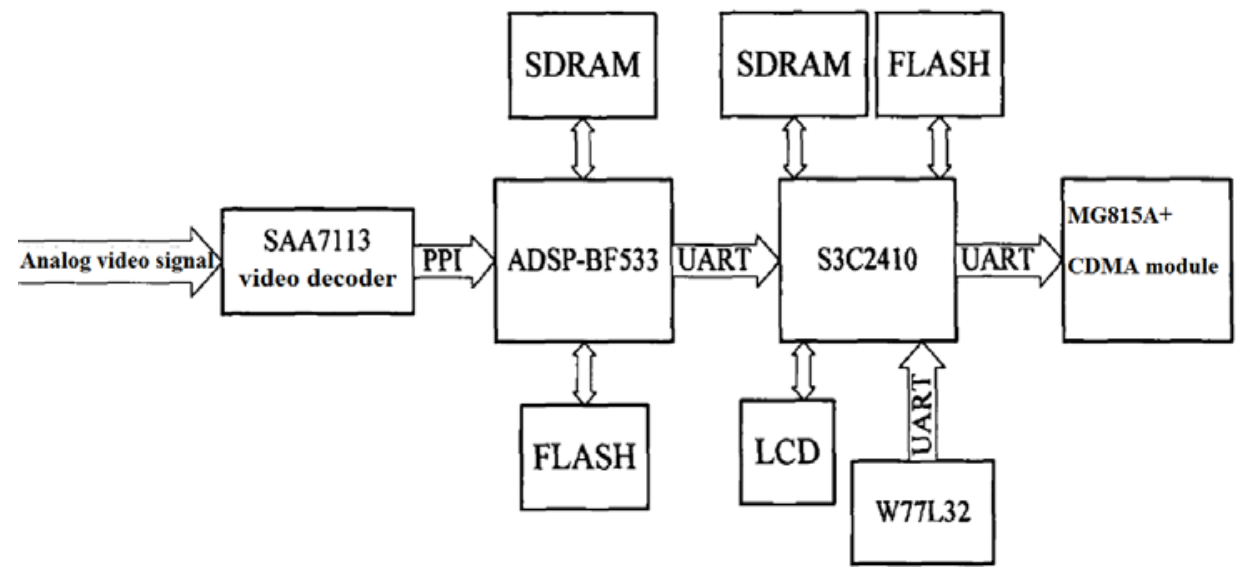

Figure 2 The hardware structure flowchart of locomotive vehicle terminal data collection system

As shown in Figure 2, the entire system incorporates ARM, DSP and 51 microcontroller and other multiple embedded processors, which separately realizes different functions in data collection system. The figure shows three components of data collection system, which are connected by three UARTs of S3C2412, to ensure no interference between operations of the various functional parts. Between them, comprehensive serial design approach is also adopted, which greatly reduces the difficulty of system development.

Design of video data collection and compression part

The main role of this part design is to help the locomotive driver to complete video collection during work, then by wireless technology conduct video compression and transmission. In order to ensure the video effect, the system uses H.264 video compression standard. Compression method needs careful calculation of the video, so it has high requirements for performance of the hardware. During design, use ADI processor, and it is advantageous in terms of the digital signal processing and calculation. For example, ADP Blackfin products can be applied to the locomotive data collection system, which include embedded video, audio and communication application 16-bit computing processors. It is characterized by low power consumption, and integrates 32-bit RISC-type instruction set and 16-bit multiply-accumulate signal microcontroller together for use. Rely on art dual-MAC signal processing engine in Blackfin processor to achieve simplicity purpose of processor instruction set, greatly reducing the overall power consumption of data collection system, and it also combines with the single-instruction multiple-data SIMD multimedia to form unique locomotive instruction set structure.

In terms of video decoding compression, mainly use typical PHILIPS video decoding chip SAA7112, able to decode and compress analog video signal recoded in camera and reverts to standard VPO digital signal. In terms of technology, SAA7112 uses CMOS technology, which realized functional application through I2C bus connecting with system, also provides two analog channels for video data compression and collection part, making the design and implementation of video collection system easier. So based on this, design and usage of SAA7112 mainly achieves the following characteristics:

a).Able to achieve two analog preprocessing channels, able to expand to four CVBS analog input channels. In terms of programmable selection, in four video input channels, each can be composed of different modes of operation with the other three channels, thus achieving the purpose of the static and automatic gain control.

b). Able to output 8-bit VPO bus, in accordance with the 4: 2: 2 format standard of YUV.

c). Able to automatically detect the field frequency of video products, to ensure frame rate of $50 \mathrm{~Hz}$ to $60 \mathrm{~Hz}$, and able to freely switch between NTSC and PAL format. Further, in the compression 
process; able to conduct internal control and processing for different input formats luminance, chrominance signals, and saturation; Considering ADI processor supports asynchronous serial UART, so based on serial port, realize connection of data transmission module, able to transmit compressed and encoded video data to the wireless module for feedback to the ground background.

\section{Design of locomotive operation data collection part}

Locomotive operation data collection is the system described in this paper, and it has similarities and differences with conventional video monitoring system. It uses time, kilometer markers and locomotive number and other data to record locomotive operation information, and mark collected video data, to facilitate monitoring terminal to inquire and manage the monitoring video in the future.

The innovation in this system is the information security comprehensive monitoring system TAX box to record locomotive kilometer mark, locomotive number and speed and other locomotive operation information. TAX box has detection communication interface, responsible for detecting all of the information referenced during locomotive operation, for example, the information of locomotive operation communication record unit and the feedback information of detection unit to the ground backstage center, etc., and locomotive operation data collection part is related with the previous case. Locomotive operation data collection part designed in this paper uses 51 microcontroller serial ports, which support multi-machine communication, and achieve locomotive operation data collection primarily through asynchronous serial UART of microcontroller. The module has two serial ports, one of which is for data collection, and the other of which is responsible for connecting with data wireless transmission system.

Design of wireless data transmission part

Wireless data transmission part is mainly the link between locomotive operation data and ground background monitoring terminal, which implements real-time monitoring and data transmission of locomotive operation status, therefore, the wireless data transmission part should be the core of vehicle terminal monitoring.

The module uses embedded technology. Wince operating system can complete data collection and connection between wireless communication network and ordinary network, and send application data to the Internet server. The most critical part of wireless data transmission part design is the control based on the CDMA module in Wince system. It can achieve control and transmission of locomotive operation data and monitoring graphics data. In this paper, we use industrial grade wireless module based on voice communication transmission and wireless data service, and it is also an important reference for shipboard monitoring system. The wireless module integrates CDMA-based baseband circuit and RF circuit, and wireless interfaces it provides are also quite rich, such as LED, USB, and charging port and so on, but generally locomotive video monitoring system uses R-UIM serial interface. Table 1 shows pin names and definitions of all parts of R-UIM serial interface.

Table 1. Pin names and function introduction of all parts based on R-UIM serial interface

\begin{tabular}{|c|c|c|}
\hline No. & Pin name & Function introduction \\
\hline 4 & UIM_DATA & UIM card input and output data port \\
\hline 2 & UIM_CLK & UIM card clock \\
\hline 8 & UIM_RST & UIM card reset signal \\
\hline 10 & V_UIM & UIM card 3V power and voltage output \\
\hline
\end{tabular}

Moreover, the industrial module also supports routing function, able to dedicated provide data transmission system for controlled wireless interface, and to control system data in the form of asynchronous serial UART. In addition, industrial embedded TCP / IP protocol stack in industrial module, able to fully support TCP, UDP and other protocols involved in the data transmission, which makes data exchange between vehicle terminal and ground background monitoring center more convenient ${ }^{[2]}$. 


\section{Design of server monitoring terminal of locomotive video monitoring system}

Server monitoring terminal is also the important communication branch system like vehicle terminal video monitoring system, as the central part of the entire video monitoring system, mainly used for communication application between background server and vehicle terminal.

We must first understand, vehicle terminal video monitoring system has two working states: activate and dormant. When vehicle terminal system is started and connected to the network, it is also connected to the server port, but at this time vehicle terminal and server do not achieve communication, and is still in the online dormant state. So if we want to activate it we must choose vehicle terminal of target locomotive through remote monitoring terminal of ground background monitoring department. When the instruction is sent, the vehicle terminal will be transferred to activation status of data transmission. Its specific design activation code is as follows:

First start the server and write function: Long JM RunServer (struct SERVER_INFO * 1pIN = NULL

Then close service function: void JM_StopServer (void);

Check connection status function:. Long JM_CheckLinkState;

Read vehicle terminal information function: long JM_GetRemoteInfo (long handle, struct REMOTE_INFO * lpRemInfo);

Start locomotive vehicle terminal video compression encoder function: void JM_ENCStart (long handle);

Stop locomotive vehicle terminal video compression encoder function: void JM_ENCStop (long handle);

In functional design of communication application of background server and vehicle terminal, we should pay attention to using some important function, which will help the remote server to complete data communication process with locomotive vehicle, to enhance the communication quality, as shown in Figure 3.

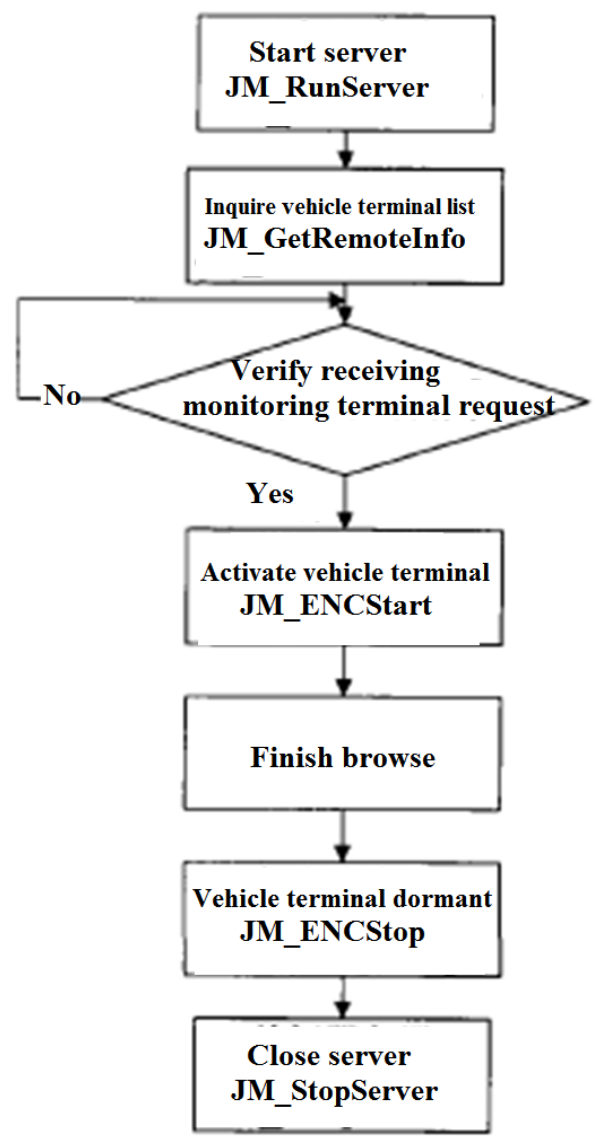

Fig. 3. Remote data communication flowchart of monitoring background server and locomotive vehicle terminal 
As shown in Figure 3, when ground background monitoring server connects and communicates with vehicle terminal, first callout JM_RunServer function to start the server, then connect with server through querying online vehicle terminal data, connect to the server, with the help of remote monitoring terminal send activation request for specified target vehicle, after verification callout again JM_ENCStart function to start vehicle compression coding system on vehicle terminal and allow vehicle terminal to be fully activated. Transmit processed video information to the remote monitoring terminal from the server, and notify the server to browse monitoring terminal information. After completion of the compression and storage of video information, vehicle terminal will be dormant again, and the server will automatically shut down, which means the communication is completed $^{[3]}$.

\section{Conclusions}

Locomotive video monitoring system is the performance supplement for locomotive operation safety and operation mechanism. It can not only assist locomotive driver with safe driving, provide more humane auxiliary services, but also help realize the connection and exchange between ground management and locomotive operation data, to achieve the purpose of remote data transmission, promote supervisory level of ground locomotive background monitoring center.

\section{References}

[1] Sui Yumin. On application of video monitoring systems in locomotive rolling stock plant. TV technology, 2009,33 (4): 82-84.

[2] Di Jingshu. On algorithm of railway locomotive intelligent video monitoring system. Harbin Institute of Technology, 2010.44-46.

[3] Deng Ji. Research and design of locomotive video monitoring system. Wuhan University of Technology, 2008.28-53. 